\title{
DOI: https://doi.org/10.24297/jap.v16i1.8460
}

\section{Study the Influence of the number of beams on radiotherapy plans for the hyopfractionated treatment of breast cancer using biological model}

\author{
Z. Mansour ${ }^{1}$, Ehab M. Attalla², A. Sarhan ${ }^{3}$, Ibrahim A. Awad ${ }^{4}$ M.I. Abdel Hamid ${ }^{5}$ \\ ${ }^{1}$ Department of Clinical Oncology\& Nuclear Medicine, Faculty of Medicine, Mansoura University, Mansoura, \\ Egypt \\ ${ }^{2}$ Department of Physics, Division of Medical Physics, National Cancer Institute, Cairo University, Egypt \\ 3, 5 Polymer Research Group, Physics Department, Faculty of Science, Mansoura University, Mansoura, Egypt \\ ${ }^{4}$ Department of Clinical Oncology and Nuclear Medicine, Faculty of Medicine, Mansoura University, Mansoura, \\ Egypt
}

Khaled_zizi12@yahoo.com, attalla.ehab@gmail.com,3,5afaf_sarhan2003@yahoo.com,awadonc@gmail.com,

\begin{abstract}
Purpose: To evaluate the biologic differences in treatment plans with different number of beams on 3D conformal radiotherapy for breast cancer patients, and compute the percent of probability to tumor control and complication of normal tissue probability using matlap program. Then determine the optimum plan through these values.
\end{abstract}

\section{Materials and Methods}

The study potentially included 13 of the female patients diagnosed with breast cancer who were treated after surgery in the Elkhir Hospital and Mansoura University (radiotherapy unit). All patient applied to a standard dose of $40 \mathrm{~Gy} / 15$ per fractions to both breast and supraclavicular. Two treatment plans were done by Prowess Panther TPS (Treatment Planning System) and changing the number of beams for each patient then dose-volume histogram (DVH) for each patient was imported to MATLAB program.

\section{Results}

All the results showed that the average TCP\% of PTV of the plans that contains more numbers of beams is 54.2 $\%$, while in other plans, $54.6 \%$, meaning that in more numbers of beams the tumor control is nearly the same as less numbers of beams. While the average NTCP of heart (normal tissue) is $23.5 \%$ with more number of beams and $25.4 \%$ with less number meaning that complication of some normal tissue such as heart is slight decrease with increasing the number of beams (less damage), but no significance value, also increasing the number of beams of the other OAR such as lung result in slight decrease in damage to normal tissue, but no significance also.

\section{Conclusion}

The numbers of beams are less important to verify control tumor and complications to normal tissue probabilities with planning proposed because the damage to normal tissue and the tumor control probability has nearly the same effect with changing the number of beams. Radio oncologist and medical physicist must make a decision about treatment though the accurate values of TCP and NTCP, and this achieved by test the accurate plan based on these values. MATLAB program is a tool to compute these values. This study avoids the trials and errors that medical physicists counteract by increasing or decreasing the number of beams, and this 
saves time to medical physicists and gives a chance to other patients to be treated, so it is very important to radiotherapy centers.

Keywords: breast cancer, number of beams, TCP: Tumor Control Probability, NTCP: Normal Tissue Complication Probability EUD: Equivalent Uniform Dose, HFRT: Hypofractionated radiotherapy, and MATLAP program.

\section{Introduction}

Breast cancer is the most common cancer among females. Its treatment includes surgical, radiotherapy (RT), and systemic treatment with chemotherapy (CT) and hormone therapy, or a combination of all these. Radiation after breast-conserving surgery (BCS) for early as well as locally advanced tumor after neoadjuvant chemotherapy (NACT) is now considered as an integral part of Breast-Conserving Therapy (BCT) whereas post-mastectomy radiotherapy (PMRT) to chest wall and or regional area is considered beneficial for a selected group of high-risk patients. PMRT decreases loco-regional recurrence (LRR) in women with operable breast cancer and enhances survival. Hypo fractionated schedule would be more appealing and convenient for patients than Conventional [1-5]. The aim of radiation oncology is the increasing the curing rates of the tumor, that typically based on the delivered dose. The distribution of dose in both the tumor and organs at risks (OAR, is calculated by specific algorithms to the dose calculation. Basing on the predicted dose distribution, radiobiological models are capable of estimating the "tumor control probability" (TCP) and "normal tissue complication probability" (NTCP). These models are depending on various statistical and mathematical concepts. Some of these models are available in the treatment planning systems (TPS) directly that are used for calculating the dose distribution. Already the effect the number of beams contributes to these values [6-12].

These aims can be described quantitatively by dose-response curves for the tumor and normal tissue, as described in figure (1). Increasing the dose to the tumor leads to increase in the tumor control probability (TCP) also. Does increasing lead to the normal tissue complication probability (NTCP), which frequently is the limiting factor in clinical situations? In the region between both two curves "denoted as therapeutic window", the probability of tumour control with no complications to normal tissue reaches a maximum at the optimum dose D opt. If type and percentage of the related complications are not accepted, however this optimized dose may not be feasible to be applied in clinical situations, and the probability for tumour control will be even lower [13] This study aiming to the evaluation of the advantage that calculations program (MATLAP) represents. It based on TCP and NTCP radiobiological models and their biological variables for their applications on daily clinic in hypofractionated radiotherapy to predict treatment planning with largest tumor control probability and minor damage probability.

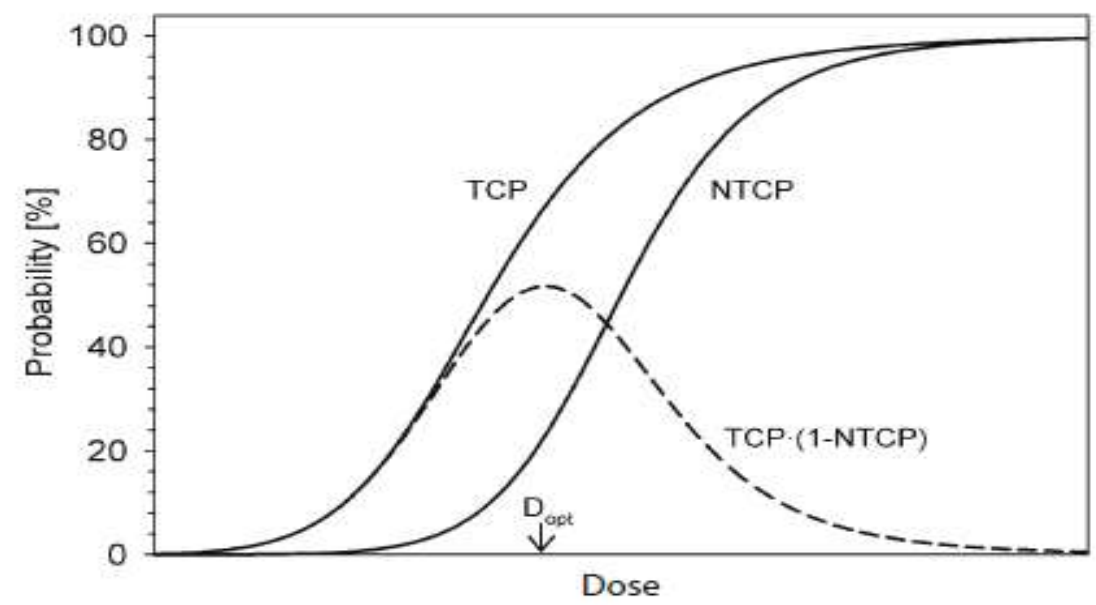

Fig. (1) Dose-response curve for tumour control probability (TCP) and normal tissue complication probability (NTCP). The maximum probability for tumour control without normal tissue complications (dashed line) is reached at the optimum dose $\left(D_{\text {opt }}\right)$ 


\section{Materials and methods}

\subsection{Materials}

\subsubsection{Prowess Panther (TPS)}

Prowess Panther TPS (Treatment Planning System) (version 7.6c, Philips Healthcare, Best, and The Netherlands) can be easily defined as Prowess Panther TPS VPS plus dose calculations. This TPS provides a cost-effective solution for CT simulation and three-dimensional therapy treatments planning system and designed to work via a fully networked department. It is designed for concurrent treatment planning that allows each member in the planning team to perform their part of the process from their workstation, at their desk, on their own schedule. The TPS simulation process provides the tools for one to design a treatment. Panther has the ability to receive information via a network system, from any manufacturer's CT scanner using DICOM 3.0 format. Using Panther, one can manipulate and consider multiple-beam geometries and the irradiated anatomy through digitally reconstructed radiograph (DRRs). The tumor volume is delineated by drawing outlines on the axial view. In the Beam's Eye View, the program simulates accurately both blocking and multi-leaf collimator blocking (MLC). These blocks are superimposed on the DRR. Advanced tools for $3 \mathrm{D}$ visualization are transverse planes and wireframe contours surrounding 3D renderings of internal tumors and structures. Following the computer simulation, the dose generated actually from the beams can be displayed in real-time. Changing the beam or plan and show the effect on the patient dosimetry, immediately [14].

\subsubsection{MATLAB}

The Math works, Inc., Natick, MA (MATLAB R2014a). This development software has served as a useful tool for processing the pencil beam data sets. MATLAB is a numeric computation and visualization soft-ware system [15].

\subsubsection{Linear accelerator}

In a linear accelerator (linac) charged particles acquire energy moving on a linear path; their characteristic feature is that particles pass only once through each of the accelerating structures. The linear accelerator used is $6 \mathrm{MV}$ or $15 \mathrm{MV}$ of the ELEKTA; Precise [16].

\subsection{Methods}

The study prospectively included 13 of the female breast cancer patients who were irradiated, after surgery in the Elkhir Hospital and Mansoura University (radiotherapy unit) between January 2018 and December 2019. Inclusion criteria: Collect host and treatment-related factors: age, histological types of the tumor, the grade of the tumor, PT stage and PN stage of the tumor according to the clinical TNM Staging System. Patients underwent breast cancer surgery in the hospital with breast cancer confirmed by postoperative pathology diagnosis age from 25 to 75 years old. All patient applied to a standard dose of ( $40 \mathrm{~Gy} / 15$ fractions/3 weeks) to both breast and supraclavicular. All the patients included in this study are listed in table 1 (a \& b) where (a) represent number of beams and (b) represent the less number of beams.

\subsection{Statistical Analysis}

The test of significance was used and considered is the T-test. The quantitative data were presented in the form of mean and standard error of mean. Significance was considered at a p-value less than 0.05 .

Table (1a) patient's treatment plans and their information, (a) represents an increasing number of beams 


\begin{tabular}{|c|c|c|c|c|c|}
\hline Patients group a & Technique & $\begin{array}{l}\text { Number of } \\
\text { fields }\end{array}$ & Diagnosis & $\begin{array}{l}\text { Age } \\
\text { (year) }\end{array}$ & $\begin{array}{l}\text { Max. } \\
\text { Dose\% }\end{array}$ \\
\hline 1 & $\begin{array}{l}\text { Tangential beams } \\
\text { with single isocenter }\end{array}$ & 8 & RT BR TXNO & 40 & 113.8 \\
\hline 2 & Half beam block & 6 & LT BR CW LN & 45 & 113.1 \\
\hline 3 & $\begin{array}{l}\text { Tangential beams } \\
\text { with single isocenter }\end{array}$ & 6 & $\begin{array}{c}\text { RTBR T2N0 } \\
\text { MRM }\end{array}$ & 64 & 112.2 \\
\hline 4 & Half beam block & 6 & $\begin{array}{c}\text { Rt BR } \\
\text { MRM T3N3 }\end{array}$ & 26 & 111.2 \\
\hline 5 & Half beam block & 6 & $\begin{array}{c}\text { Rt BR LN } \\
\text { MRM }\end{array}$ & 30 & 111.5 \\
\hline 6 & Half beam block & 6 & RTBR LN MRM & 53 & 113.5 \\
\hline 7 & $\begin{array}{l}\text { Tangential beams } \\
\text { (isocenter) }\end{array}$ & 6 & LT CW T1N0 & 50 & 112 \\
\hline 8 & Half beam block & 2 & LT BR T1N0 & 45 & 111.1 \\
\hline 9 & Half beam block & 2 & LT BR MRM & 50 & 112 \\
\hline 10 & isocenter technique & 6 & LT BR T1N0 & 50 & 115.2 \\
\hline 11 & isocenter technique & 6 & MRM RT BR NO & 50 & 115.8 \\
\hline 12 & Half beam block & 6 & T1N1M0 & 45 & 111.9 \\
\hline 13 & Half beam block & 6 & RTCW + TXN1 & 23 & 112.9 \\
\hline
\end{tabular}

Table (1b) patient's treatment plans and their information. (b) represents decrease number of beams 


\begin{tabular}{|l|l|l|}
\hline Patient group b & Number of fields & Max. dose\% \\
\hline 1 & 7 & 114.2 \\
\hline 2 & 5 & 116.6 \\
\hline 3 & 5 & 116.1 \\
\hline 4 & 5 & 111.8 \\
\hline 5 & 5 & 113 \\
\hline 6 & 5 & 113.6 \\
\hline 7 & 5 & 114 \\
\hline 8 & 3 & 111.1 \\
\hline 9 & 3 & 111.9 \\
\hline 10 & 5 & 113 \\
\hline 11 & 5 & 112.9 \\
\hline 12 & 5 & 115.9 \\
\hline 13 & 5 & \\
\hline & & \\
\hline
\end{tabular}

\subsection{Treatment protocol}

This work was designed so that it supports the analysis of DVH- and examining the breast cancer patients treated with 3D. 13 patients were treated with radiotherapy in this work. We have participated in all different diagnosis of breast cancer include mastectomy and with adenocarcinoma. Patients were treated in hypo fractionated schedules. DVHs of the treatment were imported.

Through tomographic slices was done runaround of the area where was located injury, PTV (planning Target Volume) Figure (2). DVH was built for each PTV of each patient, and organs at risk and data were imported.

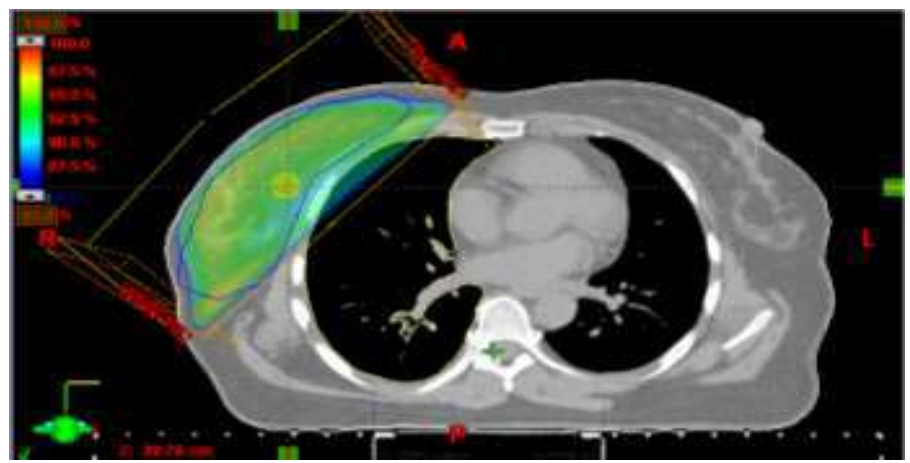

Fig. (2). Dose homogeneity in breast cancer treatment, RT lung is OAR 
Generally, in a cancer breast treatment, organs at risk "OAR" are left lung or right and heart. The technique developed in planning was standard, consisting of two tangential oblique fields including chest wall $[17,18]$. The prowess was used for 3D planning of the patients enrolled in this study. The imported patients were treated using two techniques for all target volumes. (PTV, CTV, lung, and heart) [19].The two plans are done for each patient. And the numbers of beams were changed for each of the two plans. Because different treatment plans may lead to dose distributions having similar gross dose measures (such as mean dose), but characterized by DVHs with very different shapes. To determine the optimal plan, in this case, clinicians may need to rely on relatively vague notions of dose-volume characteristics of different tissues.

Clearly, a natural application of radiobiological modeling to radiotherapy is the ranking of treatment plans through a more explicit calculation of TCP and NTCP values using models that automatically incorporate the available clinical data regarding the dose-volume characteristics of different tissues $[20,21]$ then compute the NTCP and TCP for each plan for all patients.

\subsection{Calculating the NTCP and TCP using a biological model}

The EUD (equivalent uniform dose model was used to calculate the TCP [22-24]

$$
T C P=\frac{1}{1+\left(\frac{T C D 50}{E U D}\right)^{4850}}
$$

The TCD50 is the dose to control $50 \%$ of the tumors when the tumor is homogeneously irradiated. $\bigvee 50$ describes the slope of the dose-response curve. EUD is calculated as:

$E U D=\left(\sum v_{i} D_{i}^{a}\right)^{1 / a}$

Where $(v i)$ is the fractional organ volume that receives a dose $(D i)$ and $(a)$ is a tissue-specific parameter describing the volume effect. In this study, the value of $(a)$ and other parameters TCD50 and $\gamma 50$ were taken, as listed in table (2) [25-28]. For comparative aim, the values for TCD50 and $\gamma 50$ for adjuvant radiotherapy and curative aim were investigated in order to evaluate the TCP-values with physical indices from DVH.

These equations are written in MATLAP in order to analyze the DVH for each patient using the specific program. Save this file in Matlab as eudmodel.m \%EUDMODEL (DVH), where DVH is a 2 column matrix corresponding to the cumulative, not\% differential, dose-volume histogram. The 1st column corresponds to increasing absolute dose or \%percentage dose values, and the 2 nd column to the corresponding absolute or relative volume value $\%$. The matrix must have a minimum of two rows, and both columns must be of equal length.

Table (2) radiobiological parameters used to calculate NTCP and TCP

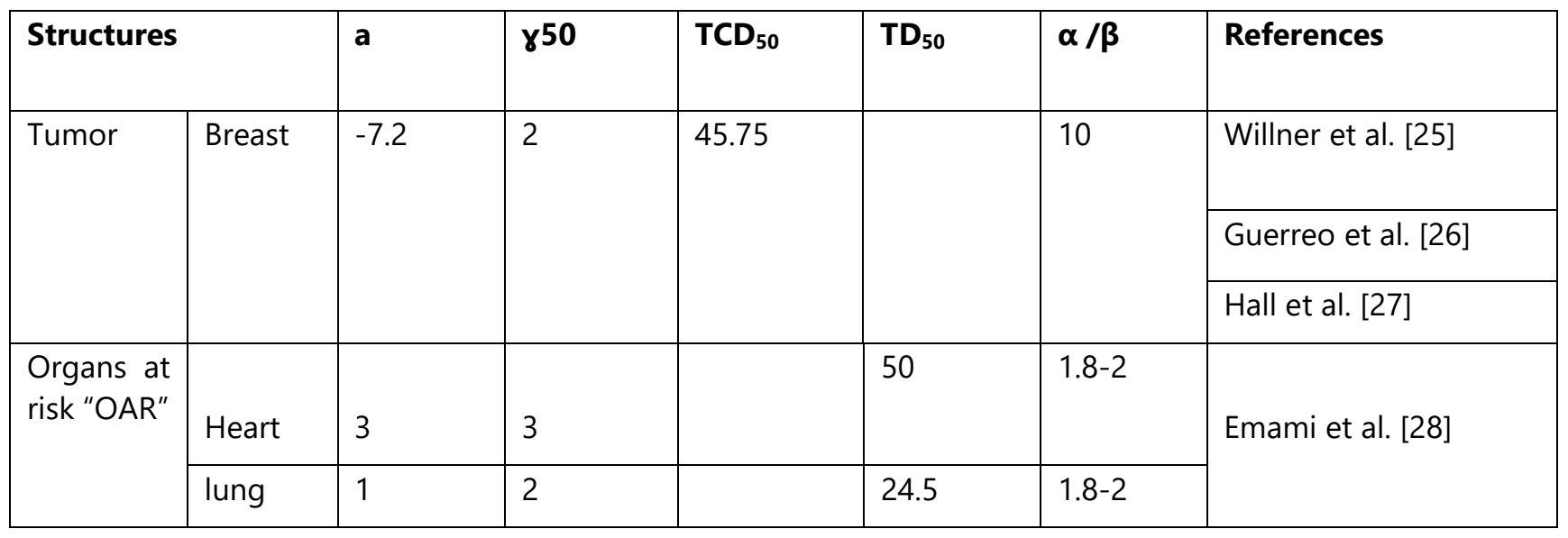

\section{Results and discussion}


The result of the MATLAP program that the user must follow the instructions is shown in Figure $3(a \& b)$. All the values of the two plans for each patient obtained are listed in table 2(a\& b), where (a) represent more number of beams and (b) represent less number of beams.

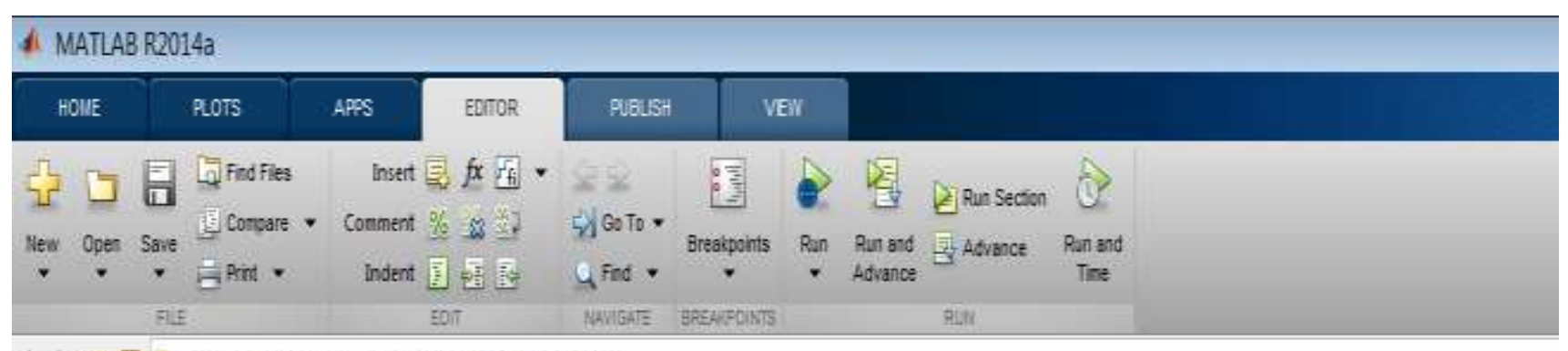

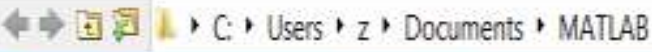

E Editor - C.users zi Desiktopleudmodel.m

eudmodelm $x+$

ASave this file in Yaclab as eudinodel.m

350DMODEL (DVH), where DVH is a 2 colum matrix corresponding to the cumlative, not

sdifferential, dose volume bistogran. The lsc colum corresponds to increasing absolute dose or

spercentage dose vaivea, and the 2 nd colum to the corresponding absolute or relative volume value.

sThe matrix mat have a minimu of two rorg, and both columns must be of equal length.

sby Hiram A. Gay, MD

\$Reriged July \& 2007

Glunction probability $=$ eudrodel (dvh)

fuser ingut section

clc; disp ('Telcame to the EquivaIent. Oniform Dose (EvD)-Based YodeI Program'); disp(" ');

disp ('Please note that: 1) the variable dvh should be a condarrve, not differential, DVH');

disp(' 2) the progran assumes that all treatment fractions are equal');

disp(' '); disp (" ');

tend of uger input section

y=input('Enter the number of structure column: '); disp(' '); disp(' ');

$s=\mathrm{y}+1$;

sverifying that the cumlative DVA has at least 2 rows and columa

$[\mathrm{nb}, \mathrm{N}]=\mathrm{size}(\mathrm{duh})$;

if $(n b<2)$

disp('Brror: Cumulative duh mast have at least 2 rows.'); return;

end

if $\{1 \mid<<\}$

disp ('Error: Cumulative dvh mast have at least 2 columns.'); return; end

kverifying that the cumlative DVH has no negative numbers in the dose or volume columns

Ffor $i=1: n b$

if $($ divh $(i, 1)<0)$

message = sprintf ('Brrox: Dose data error. drh colum 1, row \& \&g is negative', i) ;

disp (nessage) ; return;

end

if (divi $(i, s)<0)$

message = sprintf ('Errox: Volune data error. whb colum 2, rov so is negative', i) ;

disp (nessage); return;

Ready

Fig. (3a) indicate our MATLAP program to calculate TCP and NTCP 


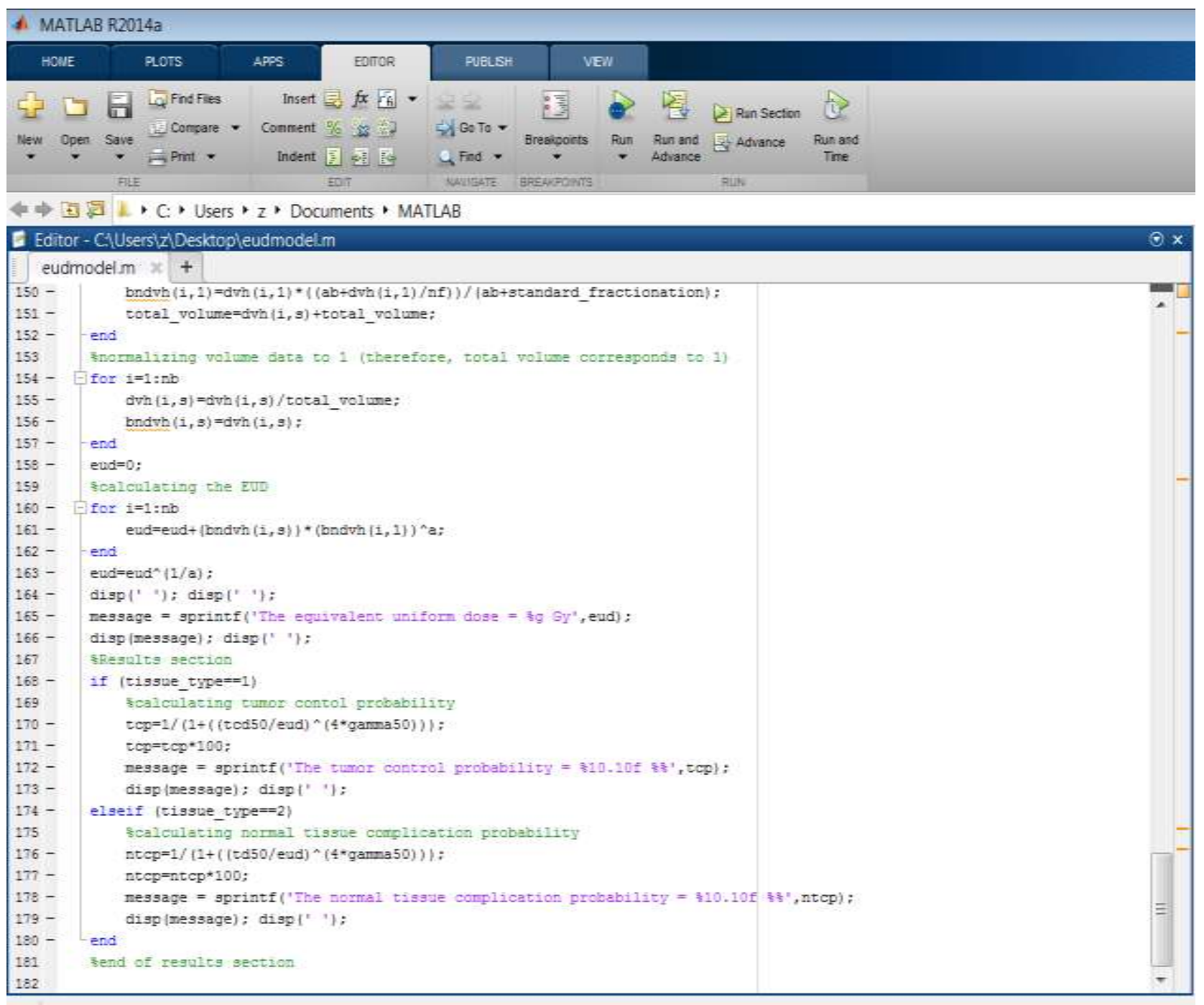

Fig. (3b) indicate our MATLAP program to calculate TCP and NTCP

Table 2 (a) shows the results of our program of the group (a) that indicate more number of beams

\begin{tabular}{|l|l|l|l|l|}
\hline $\begin{array}{l}\text { Patients group a } \\
\text { (increase number of } \\
\text { beams) }\end{array}$ & $\begin{array}{l}\text { TCP \% of } \\
\text { PTV }\end{array}$ & $\begin{array}{l}\text { NTCP \% OF } \\
\text { HEART }\end{array}$ & $\begin{array}{l}\text { NTCP \% OF } \\
\text { LUNG }\end{array}$ & $\begin{array}{l}\text { Number of } \\
\text { beams }\end{array}$ \\
\hline 1 & $52.76 \%$ & $19 \%$ & $32.92 \%$ & 8 \\
\hline 2 & $50.78 \%$ & $30.8 \%$ & $39.08 \%$ & 6 \\
\hline 3 & $48.9 \%$ & $20 \%$ & $38.7 \%$ & 6 \\
\hline 4 & $53.9 \%$ & $14 \%$ & $34.9 \%$ & 6 \\
\hline 5 & $50.07 \%$ & $20 \%$ & $40 \%$ & 6 \\
\hline 6 & $55 \%$ & $23 \%$ & $29 \%$ & 6 \\
\hline 7 & $60 \%$ & $26 \%$ & $35 \%$ & 6 \\
\hline 8 & $63 \%$ & $35 \%$ & $24 \%$ & 3 \\
\hline 9 & $53.7 \%$ & 34.9 & $16 \%$ & 3 \\
\hline
\end{tabular}




\begin{tabular}{|l|l|l|l|l|}
\hline 10 & $55 \%$ & 13.98 & $32 \%$ & 6 \\
\hline 11 & $57.69 \%$ & $8 \%$ & $14.56 \%$ & 6 \\
\hline 12 & $54 \%$ & $22 \%$ & $\% 32$ & 6 \\
\hline 13 & 50.7 & $40 \%$ & $52 \%$ & 6 \\
\hline
\end{tabular}

Table 2 (b) shows the results of our program of the group $b$ that indicate less number of beams.

\begin{tabular}{|l|l|l|l|l|}
\hline $\begin{array}{l}\text { Patients group b } \\
\text { (decrease number } \\
\text { of beams) }\end{array}$ & $\begin{array}{l}\text { TCP } \% \\
\text { of } \\
\text { PTV }\end{array}$ & $\begin{array}{l}\text { NTCP \% } \\
\text { OF HEART }\end{array}$ & $\begin{array}{l}\text { NTCP } \% \\
\text { OF LUNG }\end{array}$ & $\begin{array}{l}\text { Number of } \\
\text { beams }\end{array}$ \\
\hline 1 & $52.81 \%$ & $21.9 \%$ & $35.04 \%$ & 7 \\
\hline 2 & $53.2 \%$ & $31 \%$ & $39.06 \%$ & 5 \\
\hline 3 & $54 \%$ & $18.9 \%$ & $38.7 \%$ & 5 \\
\hline 4 & $54 \%$ & $16 \%$ & $34 \%$ & 5 \\
\hline 5 & $50.53 \%$ & $17.93 \%$ & $38 \%$ & 5 \\
\hline 6 & $54.5 \%$ & $23 \%$ & $29 \%$ & 5 \\
\hline 7 & $56 \%$ & $30 \%$ & $36 \%$ & 5 \\
\hline 8 & $63 \%$ & $35 \%$ & $24 \%$ & 2 \\
\hline 9 & $53.7 \%$ & $34.8 \%$ & $18 \%$ & 2 \\
\hline 10 & $56 \%$ & $14 \%$ & $32 \%$ & 5 \\
\hline 11 & $59 \%$ & $8 \%$ & $16 \%$ & 5 \\
\hline 12 & $53 \%$ & $40 \%$ & $52 \%$ & 5 \\
\hline 13 & $50.7 \%$ & $40 \%$ & $52 \%$ & 5 \\
\hline
\end{tabular}

All the results showed that the average TCP\% of PTV of the plans that contains more numbers of beams is 54.27 \% while in other plans $54.65 \%$. Mean \pm SEM of the group (a) is $54.27 \pm 1.121$ while in group b is $54.65 \pm 0.9297$ $\mathrm{N}=13$.

This show that there is no significance in the group (a) (increasing numbers of beams) versus group (b) (less numbers of the beam) means that the tumor control by increasing the number of beams is nearly the same as decreasing the number, $b$ value $=(0.7964)$. While the Mean \pm SEM of group (a) of NTCP of heart is $23.59 \pm$ 2.609. while in group (b) is $25.43 \pm 2.883$ meaning that complication of some normal tissue such as heart is less with increasing the number of beams there is slight decrease in complication means less damage to normal tissue but also no significance value, $p$-value $=0.6413$, also in other OAR such as Lung, NTCP in group (a) is 
average $32.3 \%$ while in group b $34.14 \%$ meaning there is slight decrease in complication probability in normal tissue with increasing the number of beams of lung also no significance value, $p$-value $=0.6621$. Figure $4(a, b$, and c) shows the effect of numbers of beams on tumor and normal tissue. Our results agree with Armando Astudillo-Velázquez, et al. within $\pm .7 \%$ [17] and KS Jothy Basu, Amit Bahl, V Subramani, et al. within \pm 5.5\% [29].

Patients have shown a ratio of TCP for both plans 0.9 , indicating that control will be the same with the error of $0.1 \%$. While the damage to normal tissue is less in increasing the number of beams such as lung and heart as shown in table 3 but no significance. It is evident from the results that MATLAP program is able to calculate the NTCP and TCP values that capable of predicting optimal plan and the number of beams has nearly no significant effect on these value, so medical physicist must test the optimum plan based on these values whatever the number of beams more or less. This saves the time of trials and errors of decreasing and increasing the number of beams. The medical physicist must concentrate on the other factors that give minor NTCP and more TCP rather than the number of fields. MAT LAP program facilitate every test to any plan. The study should also be applicable to other anatomic sites such as head and neck.

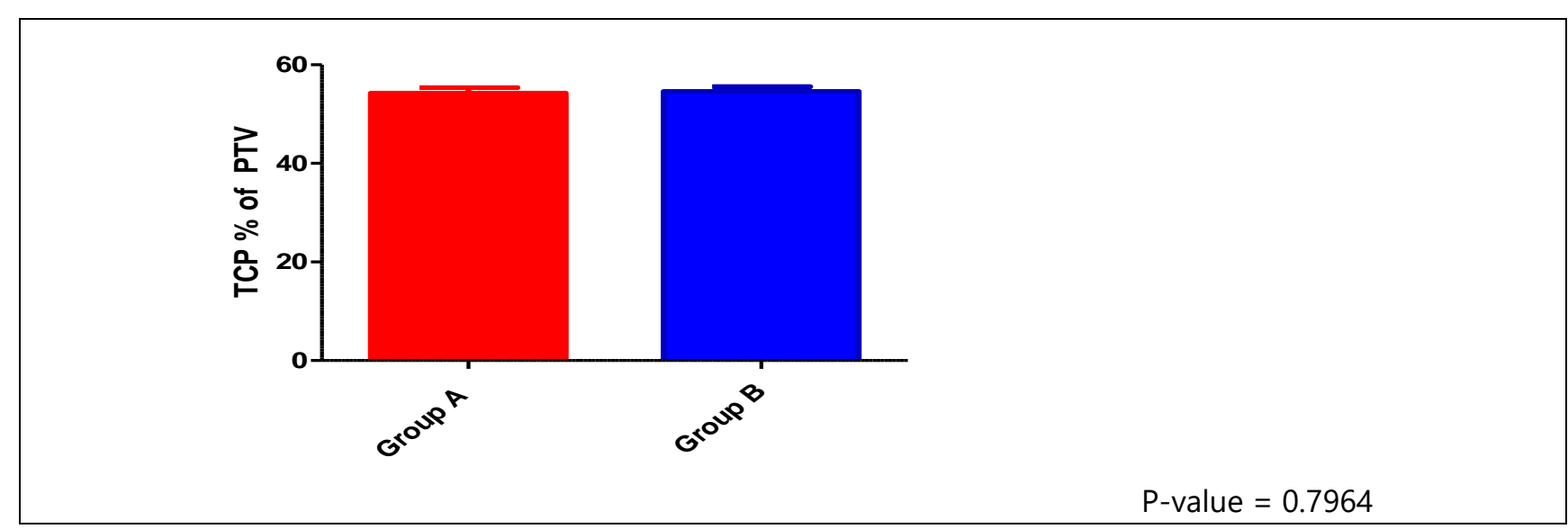

Mean \pm SEM of column A

Mean \pm SEM of column B
$54.27 \pm 1.121 \mathrm{~N}=13$

$54.65 \pm 0.9297 \mathrm{~N}=13$

Fig. (4a) show the effect of numbers of beams on tumor and normal tissue on PTV for both group a and $b$ which represent more number and less number of beams respectively

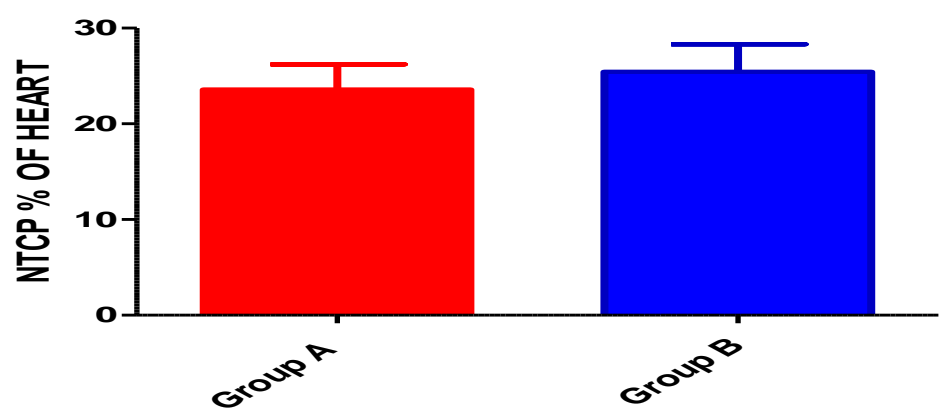

$P$ value $=0.6413$ 

Mean \pm SEM of column A
$23.59 \pm 2.609 \mathrm{~N}=13$
Mean \pm SEM of column B
$25.43 \pm 2.883 \mathrm{~N}=13$

Fig. (4 b) show the effect of numbers of beams on tumor and normal tissue on the heart for both group (a) and (b), which represent more number and less number of beams, respectively.

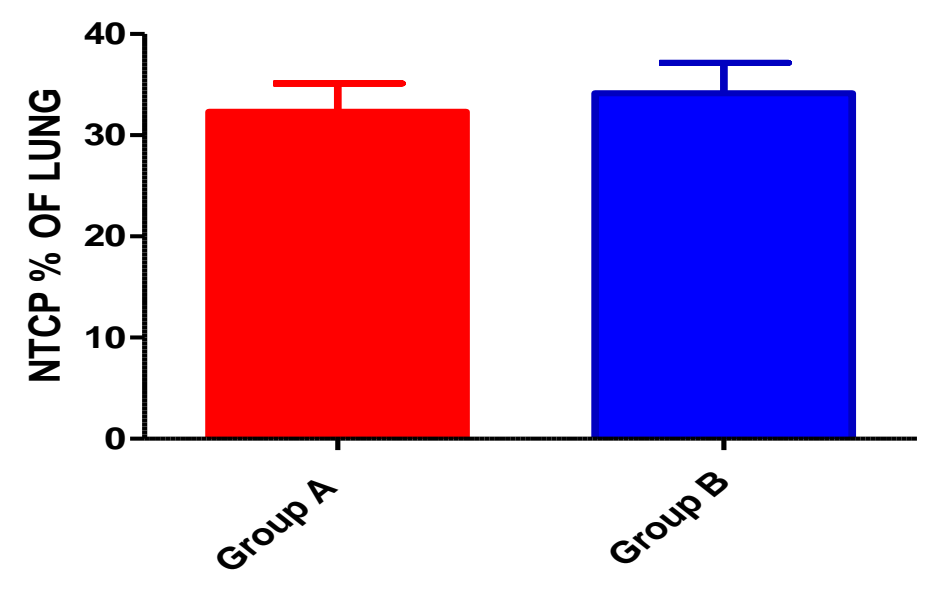

$$
\text { P-value }=0.6621
$$

$$
\begin{aligned}
& \text { Mean } \pm \text { SEM of column A } \\
& \text { Mean } \pm \text { SEM of column B }
\end{aligned}
$$$$
32.32 \pm 2.784 \mathrm{~N}=13
$$$$
34.14 \pm 3.023 \mathrm{~N}=13
$$

Fig. (4 c) shows the effect of numbers of beams on tumor and normal tissue on the lung for both group (a) and (b), which represent more number and less number of beams, respectively.

Table 3 shows the ratio of the TCP and NTCP for both more and less numbers of beams

\begin{tabular}{|l|l|l|l|}
\hline Patients & TCP ratio plan a versus plan b & $\begin{array}{l}\text { NTCP ratio of heart plan } \\
\text { a versus plan b }\end{array}$ & $\begin{array}{l}\text { NTCP ratio of } \\
\text { lung plan a vs. } \\
\text { plan b }\end{array}$ \\
\hline 1 & 0.99 & 0.86 & 0.93 \\
\hline 2 & 0.95 & 0.99 & 1 \\
\hline 3 & 0.90 & 1.05 & 1 \\
\hline 4 & 0.99 & 0.87 & 1.02 \\
\hline
\end{tabular}




\begin{tabular}{|l|l|l|l|}
\hline 5 & 0.99 & 1.1 & 1.05 \\
\hline 6 & 1 & 1 & 1 \\
\hline 7 & 1.07 & 0.86 & 0.97 \\
\hline 8 & 1 & 1 & 1 \\
\hline 9 & 1 & 1.08 & 0.88 \\
\hline 10 & 0.98 & 0.99 & 1 \\
\hline 11 & 0.97 & 1 & 0.91 \\
\hline 12 & & 0.55 & 0.6 \\
\hline 13 & 1 & 1 & 1 \\
\hline
\end{tabular}

\section{Conclusions}

This significant value leads to do separate studies to analyze the effect of each plan alone. By eudmodel.m (MATLAP program), through importing real clinical data from DVHs allows assisting to radioncologist and medical physicists in evaluation of treatment planning. It is an accessible program for everyone user. Biological constants are available in papers for the use in this program. The numbers of beams have no significance to verify control tumor and complications to normal tissue probabilities with planning proposed, the medical physicist must obtain the optimum plan based on TCP and NTCP only, and this saves the time for patients and medical physicist, so this study is extremely important to patient. The optimum plan doesn't depend on the number of beams. Radio oncologist and medical physicist must make a decision about treatment though the accurate values of TCP and NTCP, and this achieved via testing plans by MATLAP program, not by the number of beams.

\section{Acknowledgment}

We thank Mohamed Osama, Biomedical engineering and President on Medical Company, Faculty-ofengineering-Helwan-University, for his great efforts in providing me with MATLAP program and learn me how this software program work.

\section{References}

1. Amr AG and Rasha A K, (2016), "An accelerated hypofractionated schedule with a concomitant daily boost after breast conservation surgery: the feasibility and toxicity." Journal of the Egyptian National Cancer Institute; 28: [39-44].

2. Tobias JS (1996). "The role of radiotherapy in the management of cancer--an overview" Ann Acad Med Singapore.; 25: [371-379].

3. National Institute of Health Consensus Development Conference Statement (2001) "Adjuvant Therapy for Breast Cancer". J Natl Cancer Inst.; 93: [979-989].

4. American Cancer Society. Radiation Therapy. Available at:http://www.cancer.org/docroot/CRI/content/CRI 
5. Ahmed H. Oraby, Ibrahim A. Awad, Ehab M. Attalla, and Alia A. Azzam (2017)" Study the Influence of Treatment Interruptions in the Radical Irradiation of Breast Cancer journal of advances in physics; 13 ( 5): [2 3547 - $\left.34 \begin{array}{llll} & 8 & 7\end{array}\right]$.

6. Zaider M, Minerbo GN (2000) "Tumour control probability: a formulation applicable to any temporal protocol of dose delivery".journal of Phys Med Biol; 45:[279-93].

7. Bentzen SM, Tucker SL (1997) "Quantifying the position and steepness of radiation dose-response curves". Int J Radiat Biol; 71: [531-42].

8. Tucker SL, Taylor JM" Improved models of tumour cure" (1996). Int J Radiat Biol; 70: [539-53].

9. Martel MK, Ten Haken RK, and Hazuka MB. (1999 )"Estimation of tumor control probability model parameters from 3-D dose distributions of non-small cell lung cancer patients. Lung Cancer; 24: [31-7].

10. Fenwick JD, Nahum AE, and Malik ZI (2009)." Escalation and intensification of radiotherapy for stage III non-small cell lung cancer: opportunities for treatment improvement". Clin Oncol (R Coll Radiol); 21: [34360].

11. Nahum AE, Sanchez-Nieto B." Tumour control probability modeling (2001)" Basic principles and applications in treatment planning".journal of Phys Med; 17: [13-23].

12. Allen Li X, Alber M and Deasy JO (2012) "The use and QA of biologically related models for treatment planning: a short report of the TG-166 of the therapy physics committee of the AAPM" journal of Med Phys; 39: [1386-409].

13. Christian P. Karger: Biological models in treatment planning. Chapter 18: [222:235].

14. prowess Inc. treatment planning systems. Prowess panther Tps user manual. Version 5.51 website:http://www, prowess.com: page 29

15. Oldham .M, Khoo V. S., Rowbottom C. G., Bedford J. L. and Webb S. (1998).," A case study is comparing the relative benefit of optimizing beam weights, wedge angles, beam orientations and tomotherapy in stereotactic radiotherapy of the brain," journal of Phys. Med. Biol ;43: [2123-2146] .

16. Vretenar M (2014) "CERN in the Proceedings of the CAS-CERN Accelerator School: Advanced Accelerator Physics" (CERN, Geneva, 2014) .http://dx.doi.org/10.5170/CERN-2014- 009. [295:329]

17. Astudillo-Velázquez AJ. (2014) "Aplicación de los modelos radiobiológicos, TCP y NTCP, al tratamiento hipofraccionado en radioterapia, Tesis de Maestría de la Universidad Autónoma del Estado de México. México".

18. Niemierko A and Goiten M. (1991)" Calculation of normal tissue complications probabilityand dose-volume histogram reduction schemes for tissues with a critical element architecture" journal of Radiotherapy and Oncology; 20: [166-76].

19. Anil P, Kenji Myint W, Mahesh G, Sunyoung J, Jerilyn A. Logemann and Bharat B. Mittal (2010) "A computational tool for the efficient analysis of dose-volume histograms for radiation therapy treatment plans" Published in final edited form as: Journal of Appl Clin Med Phys.; 11[1]: 3013. PMC

20. Langer M., Morrill S.S, and Lane R., (1998). "A test of the claim that plan rankings are determined by relative complication and tumor-control probabilities", Int. J. Radiat. Oncol. Biol. Phys; 41 (2): [451-7]. 
21. Moiseenko V, Battista J, and Van Dyk J(2000). "Normal tissue complication probabilities: Dependence on choice of biological model and dose-volume histogram reduction scheme", Int. J. Radiat. Oncol. Biol. Phys; 46 (4): [983-93].

22. Niemierko A (1999). "A unified model of tissue response to radiation. In: Proceedings of the 41st AAPM annual meeting", Nashville, Tennessee: Med Phys [1999:1100].

23. Niemierko A (1997) "Reporting and analyzing dose distributions: a concept of equivalent uniform dose" Med Phys; 24: [103-10].

24. Gay HA, Niemierko A. (2007) "A free program for calculating EUD-based NTCP and TCP in external beam radiotherapy.; Phys Med;23: [115-25].

25. Willner J, Baier K, Caragiani E, Tschammler A, Flentje M(2002) "Dose, volume, and tumor control predictions in primary radiotherapy of non-small-cell lung cancer". Int J Radiat Oncol Biol Phys; 52: [382-89].

26. Guerrero M and Li XA (2003)" Analysis of a large number of clinical studies for breast cancer radiotherapy: estimation of radiobiological parameters for treatment planning". Phys Med Biol; 48: [3307-26].

27. Hall EJ (2000) "Radiobiology for the radiologist". 5th ed. LippincoQ Williams \& Wilkins. USA.

28. Emami B, Lyman J, Brown A, Cola L, Goitein M and Munzenrider JE (1991) "Tolerance of normal tissue to therapeutic irradiation. Int J Radiat Oncol Biol Phys, 21: [109-122].

29. Jothy KS, Amit BI, Subramani V (2008) "Normal tissue complication probability of fibrosis in radiotherapy of breast cancer: Accelerated partial breast irradiation vs conventional external-beam radiotherapy. J Cancer Res Therapy; 4 (3): [126-130]. 